\title{
OVERRINGS OF PRINCIPAL IDEAL DOMAINS
}

\section{H. H. BRUNGS ${ }^{1}$}

ABstract. All rings between a (right and left) principal ideal domain $R$ and its skewfield $Q(R)$ of quotients are quotient rings of $R$ with respect to Ore-systems in $R$.

In [4] R. Gilmer and J. Ohm investigate commutative integral domains with the $Q R$-property, i.e. with the property that every ring between the ring $R$ and its quotient field $Q(R)$ is a ring of quotients of $R$ with respect to some multiplicatively closed system in $R$. All Dedekind domains with torsion class group have the $Q R$-property.

In this note we describe all overrings (i.e. rings between $R$ and its skewfield $Q(R)$ of quotients) of a principal right and left ideal domain $R$, as left quotient rings with respect to appropriate Ore-systems in $R$. Contrary to the commutative case, we show that the left quotient ring with respect to an irreducible element need not be local. Also, a counterexample shows that for a principal left ideal domain which is not a principal right ideal domain, overrings exist which are not left quotient rings with respect to some Ore-system. See [1] for similar results.

Let $R$ be a principal left and right ideal domain. Then $R$ is a unique factorization domain, in which every nonunit element $0 \neq a$ can be written as a product of irreducible elements:

$$
a=p_{1} \cdots p_{n}, \quad p_{i} \text { irreducible for all } i .
$$

If $a=q_{1} \cdots q_{m}$ is another irreducible factorization of $a$, then $n=m$ and there exists a permutation $\pi$ of $\{1, \cdots, n\}$ such that $R / R p_{i}$ is isomorphic to $R / R q_{\pi(i)}$ as an $R$-left module for every $i([5, \mathrm{p} .34])$. We say an element $a$ is similar to $b$ in $R$, if $R / R a$ is isomorphic to $R / R b$ as a left $R$ module. It is well known, $[3$, p. 316], that $a$ and $b$ are similar if and only if there exists an element $c$ in $R$ with $R a+R c$ $=R$ and $R a \cap R c=R b c$. Similarity is an equivalence relation and leftright symmetric.

For each $a \neq 0$ in $R$ and every irreducible element $p$ in $R$ we define a nonnegative integer $h_{p}(a)=n$, if $n$ is the number of irreducible factors similar to $p$ in any irreducible factorization of $a$. It follows

Received by the editors May 4, 1970.

AMS 1970 subject classifications. Primary 16A04; Secondary 16A08.

Key words and phrases. Overrings, principal ideal domains, local rings.

1 This research was supported in part by the NRC of Canada, under Grant no. A-7254. 
that $h_{p}(a)=0$ for all $p$ if and only if $a$ is a unit in $R$. For any set $\Delta$ of irreducible elements in $R$ we define the set $S_{\Delta}=\left\{a \in R \ni h_{p}(a)=0\right.$ for all $p$ in $\Delta\}$.

We repeat the following definition: A multiplicatively closed set $M$ of regular elements of $R$ is called a (left-) Ore-system if for elements $m$ in $M$ and $r$ in $R$ there exist elements $m_{1}$ in $M$ and $r_{1}$ in $R$ such that $r_{1} m=m_{1} r$. If $M$ is an Ore-system in $R$, then there exists the ring of quotients of $R$ with respect to $M$ ([6, p. 7]). With this notation we obtain the following result:

THEOREM. Let $R$ be a left and right principal ideal domain. Then any set $S_{\Delta}$ is an Ore-system and every ring between $R$ and its skewfield of quotients $Q(R)$ can be obtained as a ring of quotients of $R$ with respect to some $S_{\Delta}$.

Proof. First we show that every $S_{\Delta}$ is an Ore-system. That $S_{\Delta}$ is multiplicatively closed follows from the unique factorization theorem stated in the beginning. It remains to show that for $s \in S_{\Delta}$ and $r$ in $R$ elements $s_{1}$ in $S_{\Delta}$ and $r_{1}$ in $R$ exist with

$$
s_{1} r=r_{1} s .
$$

Let $R s+R r=R d, s=s^{\prime} d, r=r^{\prime} d$, and it follows

$$
R s^{\prime}+R r^{\prime}=R \quad \text { for } s^{\prime} \text { in } S_{\Delta} \text { and } r^{\prime} \text { in } R \text {. }
$$

Further, $R s^{\prime} \cap R r^{\prime}=R s_{1} r^{\prime}$ and $s_{1}$ is a product of irreducible factors similar to the irreducible factors of $s^{\prime}$ and is therefore contained in $S_{\Delta}\left(\left[2\right.\right.$, p. 51]). It follows that $s_{1} r^{\prime}=r_{1} s^{\prime}$ or $s_{1} r=r_{1} s$ for $s_{1}$ in $S_{\Delta}$ and $r_{1}$ in $R$. This proves that $S_{\Delta}$ is an Ore-system and there exists an overring $S_{\Delta}^{-1} R=\left\{s^{-1} a, s \in S, a \in R\right\}$ of $R$.

Now let $T$ be any ring between $R$ and $Q(R), a^{-1} b$ an element in $T$. We may assume $b R+a R=R$, and from $b x+a y=1$, for $x, y$ in $R$, it follows that $a^{-1}$ belongs to $T$. Therefore, the inverse of every irreducible factor of $a$ is contained in $T$. It remains to show that for $p_{1}$ similar to the irreducible element $p$ in $R$ with $p^{-1}$ in $T$ we have that $p_{1}^{-1}$ is contained in $T$.

Since $p_{1}$ is similar to $p$, there exists $c$ in $R$ with $R p \cap R c=R p_{1} c$ and $R p+R c=R$. The element $c p^{-1}$ is contained in $T$ and can be written in the form $c p^{-1}=a^{-1} b$ for $a \neq 0, b$ in $R$. But then $a c=b p$ in $R p \cap R c$ and this means $a c=r p_{1} c$ or $a=r p_{1}$ for some element $r$ in $R$. From this it follows that $p_{1}^{-1}$ is contained in $T$. This proves that $T=S_{\Delta}^{-1} R$ where $\Delta$ is the set of those irreducible $q$ in $R$ such that $q^{-1}$ is not contained in $T$.

REMARK 1. Unlike the commutative case, $S_{p}^{-1} R$ is in general not a 
local ring for $\Delta$ consisting of just one irreducible element $p$. $\left(S_{p}^{-1} R\right.$ is local if and only if $h_{p}(a+b) \geqq \min \left(h_{p}(a), h_{p}(b)\right)[2$, p. 57].) For example, consider the irreducible element $p=x+t$ in the principal left and right ideal domain $R=\boldsymbol{Q}(t)[x, \sigma]=\left\{\sum_{i=0}^{n} f_{i}(t) x^{i}, f_{i}(t) \in \boldsymbol{Q}(t)\right\}$, where $Q(t)$ is the field of rational functions in one variable over the field of rationals $Q$ and $\sigma$ is the automorphism of $Q(t)$ defined by $t^{\sigma}=t+1$. Addition in $R$ is defined componentwise and multiplication by $x f(t)=f^{\sigma}(t) x$.

Now it is easy to see that $x+g(t)^{-1} g(t+1)(t+1)$ for every element $0 \neq g(t)$ in $Q(t)$, is similar to the element $x+t$ in $R$. From this we conclude that for example $S_{p}^{-1} R p$ and $S_{p}^{-1} R p_{1}$, with $p_{1}=x+$ $t^{-1}(t+1)^{2}$, are two different maximal left ideals of $S_{p}^{-1} R$ for $p=x+t$.

REMARK 2. The theorem just proved can not be extended to left principal ideal domains with maximum condition on right principal ideals, even though the rings $S^{-1} R$, as defined above, exist in this case too. Consider $Q(t)[x, \tau]=R$ as in the previous remark, but $\tau$ is now the monomorphism from $Q(t)$ into $Q(t)$ defined by $t^{r}=t^{2}$. $R$ is a principal left ideal domain with maximum condition on principal right ideals. Therefore the skewfield $Q(R)$ of quotients, $Q(R)$ $=\left\{a^{-1} b, 0 \neq a, b\right.$ in $\left.R\right\}$, exists, but the subring $T$ of $Q(R)$ generated by $R$ and $x^{-1} t x, T=R\left[x^{-1} t x\right]$, is not a ring of quotients of $R$ with respect to some Ore-system of $R$. To prove this last statement we observe first that $R\left[x^{-1} t x\right]=\left\{a x^{-1} d x b+c\right.$ for $a, b, c, d$ in $\left.R\right\}$ since $\left(x^{-1} t x\right)^{2}=t$. If we assume $T=S^{-1} R$ for some Ore-system $S$ of $R$, it follows that there exists an element $f(x)$ in $R$ of degree greater than 0 , such that $f(x)^{-1}$ is in $T$. It is easy to prove that $f(x)$ has to be equal to $u x$ for a unit $u$ in $R$ and it would follow that $x^{-1}$ is contained in $T$. But this is not possible since from $x^{-1}=a x^{-1} d x b+c, a, b, c, d$ in $R$, it follows that $1=a_{1} d x b+x c$ for some $a_{1}$ in $R$, leading to a contradiction.

\section{REFERENCES}

1. S. A. Amitsur, Remarks on principal ideal rings, Osaka Math. J. 15 (1963), 5969. MR $27 \# 1469$.

2. H. H. Brungs, Ringe mit eindeutiger Faktorzerlegung, J. Reine Angew. Math. 236 (1969), 43-66.

3. P. M. Cohn, Noncommutative unique factorization domains, Trans. Amer. Math. Soc. 109 (1963), 313-331.

4. R. W. Gilmer, Jr. and J. Ohm, Integral domains with quotient overrings, Math. Ann. 153 (1964), 97-103. MR 28 \#3051.

5. N. Jacobson, The theory of rings, Math. Surveys, no. 2, Amer. Math. Soc., Providence, R. I., 1943. MR 5, 31.

6. A. V. Jategaonkar, Left principal ideal rings, Lecture Notes in Math., no. 123, Springer-Verlag, New York, 1970.

University of Alberta, Edmonton, Alberta, Canada 